\section{Non-attendance, non-residence, non-acceptance}

Killaspy et al (2000) is one of several studies that demonstrate that non-attendance at psychiatric services is an index of increased pathology and greater need (Swofford $e t$ al, 1996; Crawford \& Wessely, 1998) and serves as a corrective to earlier suggestions that non-attenders are not usually worthy of being seen (Robin, 1976). Although the authors suggest that the first episode of non-attendance "may be an important time to intervene to attempt to prevent loss to follow-up of those with serious mental illnesses" the trends in psychiatric services are increasingly moving in the opposite direction. The growth in geographical sectors covered by community teams has many advantages (Johnson \& Thornicroft, 1993), but has naturally led to the dangers of preferentially looking after easier patients who belong within the area rather than difficult ones who might (with luck) go away if they are not seen. Although this behaviour might be considered ostrich-like in view of the fact that Killaspy et al found that patients who did not attend were more likely to be readmitted, they also noted that $27(7 \%)$ of their patients were untraceable. I suspect that most of these were extremely ill, highly geographically mobile patients who would have absorbed a significant proportion of psychiatric resources if they had been contacted proactively and who would have been seen as imposing an additional burden on services that should be primarily involved in caring for the 'real' residents in the catchment area.

We have considerable evidence of this in the inner-city area of Paddington, where there are high rates of continuous psychiatric morbidity and more than 1 in 50 of the population is referred annually (Shipley et al, 2000). This is largely because so many of the population are geographically mobile and would normally stay only briefly in the area. Even when there is intervention by the psychiatric services in the form of admission there is a five-times greater chance that such patients will be admitted to a hospital outside their area (Lamont et al, 2000) and not taken on by mainstream services. The general consequence of this is that those services that are specifically focused on the most geographically mobile population (e.g. homeless people with mental illnesses and street outreach projects) often find it difficult to arrange transfer of their patients when they eventually settle to a more permanent base because the services in the area concerned do not regard them as proper residents. I have found that patients who have transiently lived in the Paddington area are often returned there by other services on the grounds that their care belongs in the area and the patients wish to return.

In practice it is unlikely that the sound recommendation of Killaspy et al that services be more active in seeing non-attenders would be followed because it is likely to lead to the growth of imported psychopathology of severe mental illness and increased psychiatric morbidity in the areas concerned. We have recommended elsewhere (Lamont et al, 2000) that the best way forward in tackling this problem is to create regional teams not preoccupied with catchment area boundaries, who could provide consistent and appropriate care for this forgotten non-attending population.

\section{Crawford, M. J. \& Wessely, S. (1998) Does initial} management affect the rate of repetition of deliberate self harm? A cohort study. British Medical Journal, 317 985.

Johnson, S. \& Thornicroft, G. (1993) The sectorisation of psychiatric services in England and Wales. Social Psychiatry and Psychiatric Epidemiology, 28, 45-47.

Killaspy, H., Banerjee, S., King, M., et al (2000) Prospective controlled study of psychiatric out-patient non-attendance. Characteristics and outcome. British Journal of Psychiatry, I76, 160-165.

Lamont, A., Ukoumunne, O., Tyrer, P., et al (2000) The geographic mobility of severely mentally ill residents in London. Social Psychiatry and Psychiatric Epidemiology, in press.

Robin, A. (1976) Rationing out-patients: a defence of the waiting list. British Journal of Psychiatry, 129, 138-|4|.

Shipley, K., Hilborn, B., Hansell, A., et al (2000) Patient satisfaction: a valid index of quality of care in a psychiatric service. Acta Psychiatrica Scandinavica, I0I, 330-333.

Swofford, C. D., Kasckow, J. E., Scheller Gilkey, G., et al (1996) Substance use: a powerful predictor of relapse in schizophrenia. Schizophrenia Research, 20, 145-151.

P.Tyrer Division of Neuroscience and Psychological Medicine, Imperial College School of Medicine, St Mary's Campus, Paterson Centre, 20 South Wharf Road, London W2 IPD

\section{Cigarette smoking in patients with schizophrenia}

We read the article by McCreadie \& Kelly (2000) with interest. The study indicates a high rate of cigarette smoking in patients with schizophrenia. It concluded that typical patients who smoke return $18-31 \%$ of their state benefits to the Treasury in the form of taxes on the purchase of cigarettes. However, the authors have not taken into consideration the cost of smoking-related diseases such as ischaemic heart disease and chronic obstructive airways disease. Inevitably, patients suffering from such diseases require expensive cardiorespiratory investigations and medication, which results in substantial expenditure by the National Health Service. Thus, perhaps the Treasury would be better off if patients did not smoke.

A review article by Felker et al (1996) has outlined various studies which indicate increased morbidity in psychiatric patients due to various medical conditions, including cardiovascular and respiratory problems. It is difficult to show the extent to which these diseases are caused by cigarette smoking, but we all know that there is a strong association between cigarette smoking and cardiorespiratory problems.

Felker, B., Yazel, J. \& Short, D. (1996) Mortality and medical comorbidity among psychiatric patients: a review. Psychiatric Services, 47, 1356-1363.

McCreadie, R. G. \& Kelly, C. (2000) Patients with schizophrenia who smoke. Private disaster, public resource. British Journal of Psychiatry, 176, 109.

P. Arshad Calderstones NHS Trust, Mitton Road Whalley BB7 9PE

S. A. Arshad Northern General Hospital Sheffield

McCreadie \& Kelly (2000) highlight the enormous financial cost of cigarette smoking to patients with schizophrenia and extrapolate from their data that those who smoke return $18-31 \%$ of their benefits to the Treasury, thus substantially contributing to the cost of their care. We directly measured the cost of smoking to a group of patients in Waterford and found that those who smoke spend an average of $29 \%$ of their income on cigarettes (McDonald \& Sheppard, 1996). They thus contributed $24 \%$ of their income back to the Treasury in Ireland through this source alone, a proportion more akin to income tax for most people. This confirms the calculations of McCreadie \& Kelly.

Aside from a curiously reduced risk of lung cancer (Gulbinat et al, 1992), patients with schizophrenia have increased mortality from heart and lung disease (Mortenson $\&$ Juel, 1993) and it is likely that cigarette smoking contributes largely to this. In addition to such adverse health effects, cigarette 